\title{
Cyclopentanone as alternative linking reactant for heterogeneously catalysed furfural aldol condensation
}

\author{
J. Cueto, L. Faba, E. Díaz, S. Ordóñez ${ }^{\star[a]}$
}

\begin{abstract}
The use of cyclopentanone, instead of acetone, in the synthesis of diesel precursors via furfural aldol condensation is proposed in this paper. This reaction is catalyzed by a $\mathrm{Mg}-\mathrm{Zr}$ mixed oxide. In order to optimize the $\mathrm{C} 15$ selectivity, different temperatures $(293,303,313$ and $323 \mathrm{~K})$ and initial reactant rates furfural:cyclopentanone $(1: 1,3: 1,5: 1$ and 10:1) were tested. At the optimum conditions, yields for the desired $\mathrm{C} 15$ adduct are higher than $60 \%$, at reaction times lower than 4 hours, and working at mild conditions (303 K).
\end{abstract}

Biomass catalytic transformations are the most feasible alternatives for developing renewable and drop-in transportation fuels. Different processes are nowadays being considered for transforming the lignocellulosic fraction into several platform molecules, raw materials to fine chemicals as well as diesel or gasoline drop-in substitutes. ${ }^{[1]}$ The process firstly proposed by Dumesic and co-workers to transform aldehydes derived from (hemi)cellulosic biomass, furfural (FFL) and 5-hydroxylmethylfurfural (5-HMF) into diesel is one of the most promising strategies. ${ }^{[2]}$

The key step in this process is the aldol condensation, reaction that allows to increase the carbon chain length. Acetone is usually used as linking molecule in this reaction. The aldol condensation of acetone and furfural has been deep studied using different homogeneous and heterogeneous catalysts, highlighting different hydroxides $\left(\mathrm{NaOH} \text { and } \mathrm{Ca}(\mathrm{OH})_{2}\right)^{[3]}$ and mixed oxides such as $\mathrm{Mg}-\mathrm{Zr}$ or $\mathrm{Mg}-\mathrm{Al} .{ }^{[4]}$ However, the use of acetone is under controversy because of different reasons. Firstly, this molecule is traditionally obtained from the petroleum as side product in the cumene process, ${ }^{[5]}$ so the derived diesel is not entirely renewable. The main green route to obtain the acetone is the ABE fermentation, but the selectivity of this process is very low because the main scope of this reaction is to improve butanol yields. ${ }^{[6]}$ On the other hand, using acetone, the range of carbon chain length that can be obtained, C8-C13 or C9-C15 depending on the considered aldehyde, ${ }^{[7]}$ can be not enough to guarantee a good quality diesel. These restrictions justify the aim of this work, proposing the use of cyclopentanone (CPO) as a substitute of acetone for the aldol condensation with furfural.

CPO is a cyclic ketone obtained from the sugars when the dehydration (process used to obtain the furfural and $5-\mathrm{HMF}^{[8]}$ ) is combined with a selective hydrogenation. Using the optimum conditions, large cyclopentanone yields can be obtained. ${ }^{\left[{ }^{[9]}\right.} \mathrm{CPO}$ has a high potential as a versatile precursor of drugs, fragrances and insecticides. ${ }^{[10]}$ Besides, it has two carbon atoms with $\alpha$ protons, being possible to be considered as good linking molecule for aldol condensation reactions. In fact, some studies suggest this reaction to obtain compounds with longer carbon chains. ${ }^{[11]}$ The use of this molecule instead of the acetone in the furfural-acetone cross-condensation would have significant advantages. Firstly, diesel obtained can be entirely considered as renewable, because both reactants are produced from (hemi)cellulosic biomass. Moreover, the condensation adducts would have longer carbon chains (C10-C15 when furfural is used and $\mathrm{C} 12-\mathrm{C} 17$ when $5-\mathrm{HMF}$ is the aldehyde). As a consequence, the quality of this fuel would be higher. ${ }^{[12]}$ The enolization of CPO is stabilized by the inductive effect of the side carbons, so the aldol condensation should be enhanced and the retro-aldolization minimized. Finally, the total hydrogenation of these products would produce branched derivatives, increasing the quality as gasoline substitutes. ${ }^{[13]}$

Despite these potential advantages, the study of this alternative is still in the beginning stages. To the best of our knowledge, the only study of this reaction published so far is focused on the preliminary analyses of this condensation catalyzed by homogeneous catalyst $(\mathrm{NaOH}) .{ }^{[14]}$ Promising results were reported, with more than $95 \%$ of the second adduct yield (C15) in less than 2 hours. However, there are several technical, economic and environmental disadvantages of using homogeneous catalysts. ${ }^{[15]}$ Consequently, the study of this reaction using heterogeneous catalysts is essential to match this to the Sustainable Chemistry principles.

The aim of this article is to determine the feasibility of performing furfural cyclopentanone cross-condensation at mild conditions using heterogeneous catalysts and analyzing the reaction mechanism as function of the obtained data Specifically, the catalyst providing the best behavior for acetonefurfural cross-condensations ( $\mathrm{Mg}-\mathrm{Zr}$ mixed oxides) has been used as catalyst. ${ }^{[16]}$ Besides these good previous results, this material was also chosen by Liang et al. ${ }^{[17]}$ in the first approach to the cyclopentanone aldolization using heterogeneous materials, obtaining promising results in the cyclopentanone self-condensation.

\footnotetext{
Cueto, Dr. L. Faba, Dr. E. Díaz, Prof. S. Ordóñez

Department of Chemical and Environmental Engineering

University of Oviedo

C/ Julián Clavería, s/n-33006-Oviedo-Asturias (Spain)

sordonez@uniovi.es
}

Supporting information for this article is given via a link at the end of the document. 

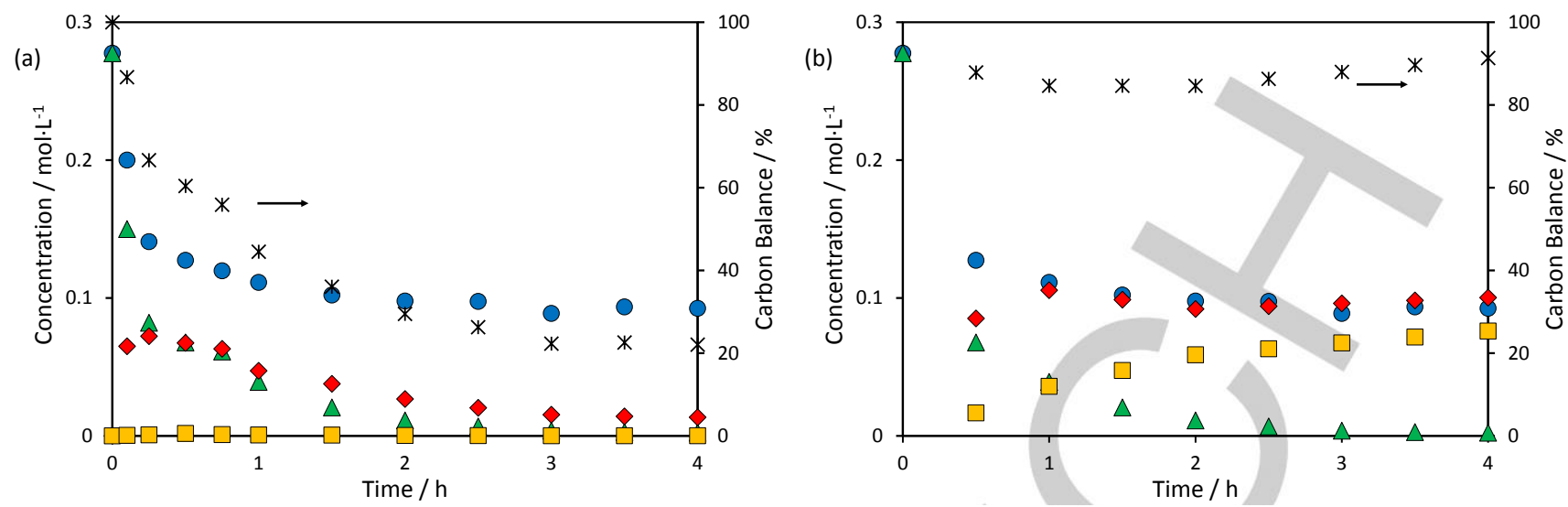

Figure 1. Time evolution of the cyclopentanone-furfural condensation ( $5 \%$ org, $1: 1)$ catalyzed by $0.5 \mathrm{~g}$ of $\mathrm{Mg}-\mathrm{Zr}$ at $323 \mathrm{~K}$ considering (a) just the liquid phase;

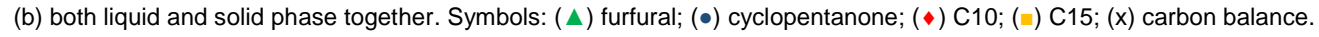

Figure 1 shows the results obtained with an equimolar ratio of both reactants (5\% of organics in water) at $323 \mathrm{~K}$ and using $0.5 \mathrm{~g}$ of $\mathrm{Mg}-\mathrm{Zr}$ catalyst. These are the previously optimized conditions for the cross-condensation of acetone and furfural ${ }^{[16]}$. A previous experiment in absence of $\mathrm{Mg}$ - $\mathrm{Zr}$ confirmed that there is not any reaction without catalyst (less than $1 \%$ of conversion after 24 hours of reaction). As it is observed in Fig. 1, there is a fast decrease in the concentration of both reactants, reaching complete conversion of FFL (limiting reactant) in less than 3 hours, with almost $68 \%$ of CPO conversion at the same reaction time. Concerning to the liquid products, seven main peaks are identified in the GC spectra. Four of them correspond to molecules with 10 carbon atoms and the other three to C15 molecules. Two peaks related to molecules with 10 carbon atoms were identified as the cis and trans isomers of the adduct obtained by the reaction of one molecule of CPO and one of FFL. In all the cases, the trans isomer signal is the most intense, in good agreement with the higher stability of this isomer. It was previously reported that the trans isomer is needed to stabilize the reaction and promote the second step of the aldol condensation. ${ }^{[16]}$ The GC-MS spectra of the other two peaks of C10 compounds were very similar to the previous one but with the presence of a water molecule in their structure. According to the mechanism proposed to other aldol condensations, ${ }^{[18]}$ these products are the adducts obtained before the dehydration step. Taking into account the scope of this reaction (to obtain fuel precursor that will be hydrodeoxygenated to the corresponding linear alkanes), all these $\mathrm{C} 10$ compounds are considered together in the analyses. Regarding to the $\mathrm{C} 15$ peaks, ${ }^{13} \mathrm{C}$ NMR analysis (Figure S1) confirms the correspondence with the compound obtained by the interaction of one $\mathrm{C} 10$ molecule with a second FFL unit being located the CPO at the central position, discarding any possible oligomerization of the reactants. In this case, no signals of the hydroxylated $\mathrm{C} 15$ precursor were detected in significant amounts. The J-coupling displacements obtained confirm the co-existence of three different isomers: ciscis, cis-trans, and trans-trans, being the last one the main one (in good agreement to be the most stable one). As in the case of the $\mathrm{C} 10$, all the $\mathrm{C} 15$ peaks will be analyzed as one. As to the products in liquid phase, the concentration of $\mathrm{C} 10$ reaches a maximum after 0.25 hours (corresponding to an atomic yield of $27 \%$ ) whereas its subsequent decrease does not match with a relevant increase in the second adduct (C15), adduct with atomic yield always lower than $2 \%$. No side-products were detected in the liquid phase (including the cyclopentanone self-condensation adducts). These results contrast with the evolution of carbon balance (lower than $50 \%$ after 1 hour and with a continuous decreasing profile to values around $20 \%$ after 4 hours).

This performance is explained because of the appearance of a high amount of a new yellow solid phase clearly segregated from the solid catalyst. This solid phase was recovered by filtration, dried, completely dissolved in ethyl acetate and analyzed by GC-FID, observing only the same peaks as those detected in the liquid phase analyses (C10 and C15 adducts). Results considering both phase together are plotted in Figure 1.b, obtaining much more congruent profiles of reaction products and carbon balance. The goodness of these analyses is clearly observed by the carbon balance, obtaining values always higher than $87 \%$. These values are in the same order of magnitude than others previously reported for biomass derived processes ${ }^{[19]}$ As to the products, C10 shows a typical profile of a first intermediate product, reaching a maximum after one hour $\left(0.11 \mathrm{~mol} \cdot \mathrm{L}^{-1}\right)$ and keeping this concentration almost constant during all the reaction. The $\mathrm{C} 15$ follows a continuously increasing profile, typical of a final product, reaching a value of $0.076 \mathrm{~mol} \cdot \mathrm{L}^{-1}$ at the end of the reaction. Final values corresponds to atomic yields of 36.2 and $41.1 \%$ of $\mathrm{C} 10$ and $\mathrm{C} 15$, respectively. Considering both condensation products together, more than $77 \%$ of final yield was obtained, being these results comparable with previous yields reported for similar reactions. ${ }^{[20]}$ However, the ratio $\mathrm{C} 15 / \mathrm{C} 10$ is not so high (1.2), indicating a product mixture that would not guarantee a good quality as diesel precursor. As a consequence, different reaction conditions (temperature and initial reagent rate) were tested trying to improve this ratio. Besides, the catalytic amount was reduced to $0.1 \mathrm{~g}$ in order to prevent the complete furfural conversion. 
In all the cases, similar profiles were obtained (included as supplementary information in the Figure S2), being congruent with the profiles shown in Fig. 1. In order to make easier the comparison, final results are summarized in Table 1, including also data obtained in the solid analyses. The influence of temperature in the solubility of these compounds was discarded by particular analyses of same solid dissolved at different temperatures, obtaining less than $0.3 \%$ of difference between $\mathrm{C} 10$ and $\mathrm{C} 15$ solubility at 293 and $323 \mathrm{~K}$. On the other hand, the influence of partial catalytic deactivation by metal leaching was discarded by ICP-MS analyses, obtaining less than $6.5 \mathrm{ppm}$ of $\mathrm{Mg}$ and $20 \mathrm{ppb}$ of $\mathrm{Zr}$ in the liquid phase. As consequence, different values obtained in these studies are only justified by the influence of the temperature. As it can be observed in Table 1, final conversions are not strongly dependent on reaction temperature, reaching always complete conversion of furfural after 4 hours and conversion higher than $65 \%$ of CPO in all the cases. The relevance of this parameter is mainly related to the time to reach this complete conversion, decreasing from 3.5 hours at $293 \mathrm{~K}$ to less than $1.5 \mathrm{~h}$ at $323 \mathrm{~K}$ (temporal profiles in Fig. S2). Concerning to the carbon balance, when only the liquid phase is considered, there is a fast decrease in all the experiments, being more relevant at highest temperatures, reaching final values around $20 \%$ at 313 and $323 \mathrm{~K}$ and to $35 \%$ at 293 and $303 \mathrm{~K}$ at the end of the reaction, being the differences again more evident at short times (values lower than $50 \%$ after only 1 hour when working at 313 and $323 \mathrm{~K}$, whereas this values is around $80 \%$ at same time when working at lower temperatures).

Once the solid phase is also considered in the analyses, the final carbon balance increases to $62 \%$ in the most adverse conditions (the highest temperature). As to the products, maximum yields were obtained at $303 \mathrm{~K}(66 \%$ considering both $\mathrm{C} 10$ and $\mathrm{C} 15$ together and with a carbon balance of $84 \%$ ), obtaining poorer values at higher temperatures. This fact is more evident in the case of $\mathrm{C} 15$, obtaining a yield of only $23 \%$ at $323 \mathrm{~K}$. At these conditions, the carbon balance also decreases $(62 \%)$, suggesting the presence of side reactions of oligomerization promoted by this second adduct. These oligomers are hydrophobic and are recovered with the main products in the solid phase. This hypothesis was corroborated by analyzing by thermogravimetry the solids recovered after the reaction. Figure 2 shows the comparison between evolutions obtained when reaction is carried out at 303 and $323 \mathrm{~K}$.

In addition to the peak corresponding to adsorbed water, three main peaks are observed in all the cases, the area of each peak being the most important differences between them. The decomposition temperatures of these peaks correspond to 535, $580-600$ and $713 \mathrm{~K}$, respectively. According to the empiric method improved by Stein and Brown, ${ }^{[21]}$ two first peaks are assumed as the volatilization of $\mathrm{C} 10$ and $\mathrm{C} 15$ main products (theoretical boiling temperatures of 530 and $590 \mathrm{~K}$, respectively), with the slight differences being attributed to the different isomers. The weight loss of these peaks is higher in the experiment at $323 \mathrm{~K}$, being congruent with the high activity at this temperature, and the worst carbon balance closure. The third peak $(713 \mathrm{~K})$ could be assigned to the $\mathrm{C} 15$ hydroxyl intermediate obtained before the dehydration that produces the condensated $\mathrm{C} 15$ (the empiric method suggests $709 \mathrm{~K}$ for this compound). This product is not detected in the liquid phase, suggesting that it remains in the solid phase or entirely adsorbed on the catalytic surface. The highest intensity of this peak at the lowest temperature indicates that a minimum of temperature is needed to produce these dehydrations to obtain the final $\mathrm{C} 15$ and the high amount of oxygen atoms justifies the so high adsorption that prevents its detection in the liquid phase. The presence of these oxygen atoms increases its solubility (considering the empirical correlations proposed by Stein and Brown ${ }^{[21]}$ the solubility of the final $\mathrm{C} 15$ is $<10^{-10} \mathrm{~mol} \cdot \mathrm{L}^{-1}$, whereas

Table 1. Results (liquid and solid phase) after 4 hours of condensation between furfural and cyclopentanone (1:1) catalyzed by $0.1 \mathrm{~g}$ of $\mathrm{Mg}-\mathrm{Zr}$ at different temperatures. ${ }^{[a]}$

\begin{tabular}{cccccc}
\hline $\mathrm{T}(\mathrm{K})$ & $\begin{array}{c}\text { Conv. FFL } \\
(\%)\end{array}$ & $\begin{array}{c}\text { Conv. CPO } \\
(\%)\end{array}$ & $\begin{array}{l}\Psi_{\mathrm{C} 10} \\
(\%)\end{array}$ & $\begin{array}{l}\Psi_{\mathrm{C} 15} \\
(\%)\end{array}$ & $\begin{array}{c}\text { C.B. } \\
(\%)\end{array}$ \\
\hline 293 & 97.0 & 69.0 & 31.6 & 19.4 & 68.1 \\
303 & 98.3 & 66.4 & 31.7 & 33.9 & 83.3 \\
313 & 99.7 & 67.6 & 28.2 & 23.1 & 67.6 \\
323 & 99.9 & 69.2 & 23.7 & 22.6 & 61.8 \\
\hline
\end{tabular}

[a] The mathematical expressions of atomic yields and carbon balance are detailed in the experimental section.

the solubility of the alcohol intermediate is $0.0519 \mathrm{~mol} \cdot \mathrm{L}^{-1}$ ) suggesting that this compound should appear in higher amount in the liquid phase. However, considering the presence of medium-strength acid-base sites of this catalyst, there is a strong interaction between the surface and the hydroxyl functional groups (mainly based on the higher polarity of this oxygen atom in comparison with the corresponding carbonyl one). As consequence and considering that this molecule has one carbonyl group and two alcohols, it can be concluded that this intermediate is strongly adsorbed. Besides, the lowest carbon balance observed in the reaction at $293 \mathrm{~K}$ can be the consequence of shift of the adsorption equilibria at the lowest temperatures. Despite the fact that $\mathrm{Mg}-\mathrm{Zr}$ is white, spent catalysts recovered after the TG analyses are slightly grey and dark grey, for reactions at 303 and $323 \mathrm{~K}$, respectively. These colors are related to the presence of oligomers (much more relevant at the highest temperature) that are stable at the maximum temperatures reached by the thermogravimetry, being

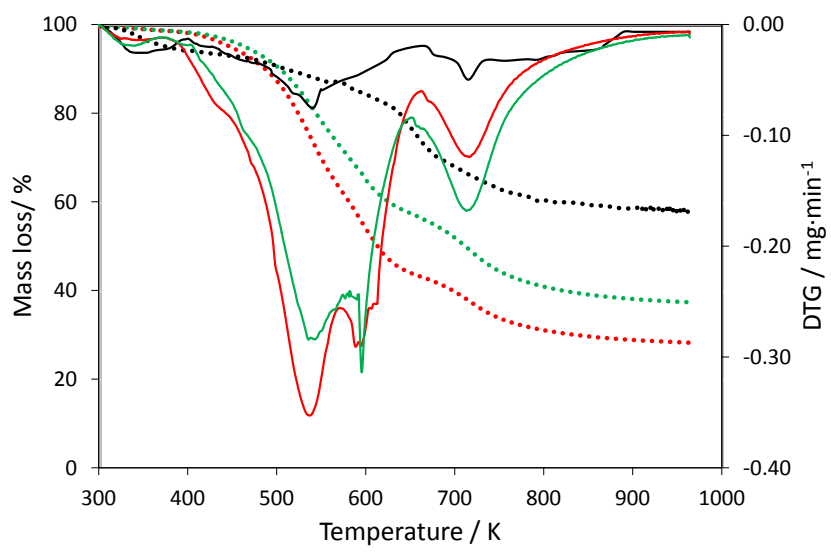

Figure 2. Thermogravimetric profiles (continuous lines are related to the mass loss whereas dotted lines are related to the DTG) obtained for the $\mathrm{Mg}-\mathrm{Zr}$ recovered after 4 hours of cyclopentanone-furfural condensation at $303 \mathrm{~K}$ (green) and $323 \mathrm{~K}$ (red), as well as the sample obtained after the solid extraction with ethyl acetate of reaction at $303 \mathrm{~K}$ (black). 
not possible to identify and justifying the final carbon balances. It highlights that no self-condensation of CPO is detected at tested conditions. This fact is opposite to results reported by Hronec and co-workers but it can be explained considering the hypothesis that furfural is strongly adsorbed on the catalytic surface, hindering the adsorption of the linking molecule. ${ }^{[16]}$ At this point, acetone self-condensation was observed in the ACEFAL condensation, ${ }^{[16]}$ but acetone presents largely lower molecular size, which allows its adsorption. In good agreement, the initial ratio between furfural and cyclopentanone conversion in heterogeneous system is 28.6 , whereas values of 1.7 are reported working in homogeneous conditions. ${ }^{[14 a]}$ These differences are in good agreement with a significant lower consumption of cyclopentanone in side reactions when working with $\mathrm{Mg}-\mathrm{Zr}$. experimental results with the previous ones reported for the condensation between furfural an acetone.

$$
r_{\text {condensation }}^{0}=\frac{1}{S_{G}} \cdot\left[\frac{d X}{d\left(\frac{t \cdot W}{n^{0}}\right)}\right]_{\left(\frac{t \cdot W}{n^{0}}\right)=0}
$$

Where " $S_{G}$ " is the surface area of the catalyst $\left(74 \mathrm{~g} \cdot \mathrm{m}^{-2}\right.$ for this $\mathrm{Mg}-\mathrm{Zr}$, as it was previously reported ${ }^{[22]}$ ); " $X$ " the limiting reactant conversion (CPO or acetone as function of the reaction); " $\mathrm{t}$ " the reaction time in minutes; "W" the mass of MgZr in grams; and " $\mathrm{n}$ ", the initial moles of CPO or acetone. Experimental results indicate that the reaction using cyclopentanone as linking molecule is more than 320 times faster than the corresponding condensation with acetone $\left(87.3\right.$ and $0.27 \mu \mathrm{mol} \cdot \mathrm{min}^{-1} \cdot \mathrm{m}^{-2}$, with

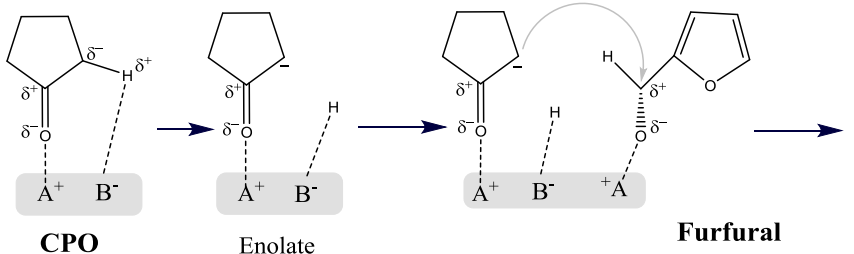

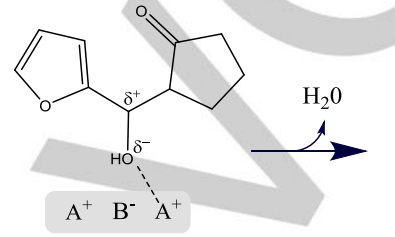

$\mathrm{C}_{10}-\mathrm{OH}$
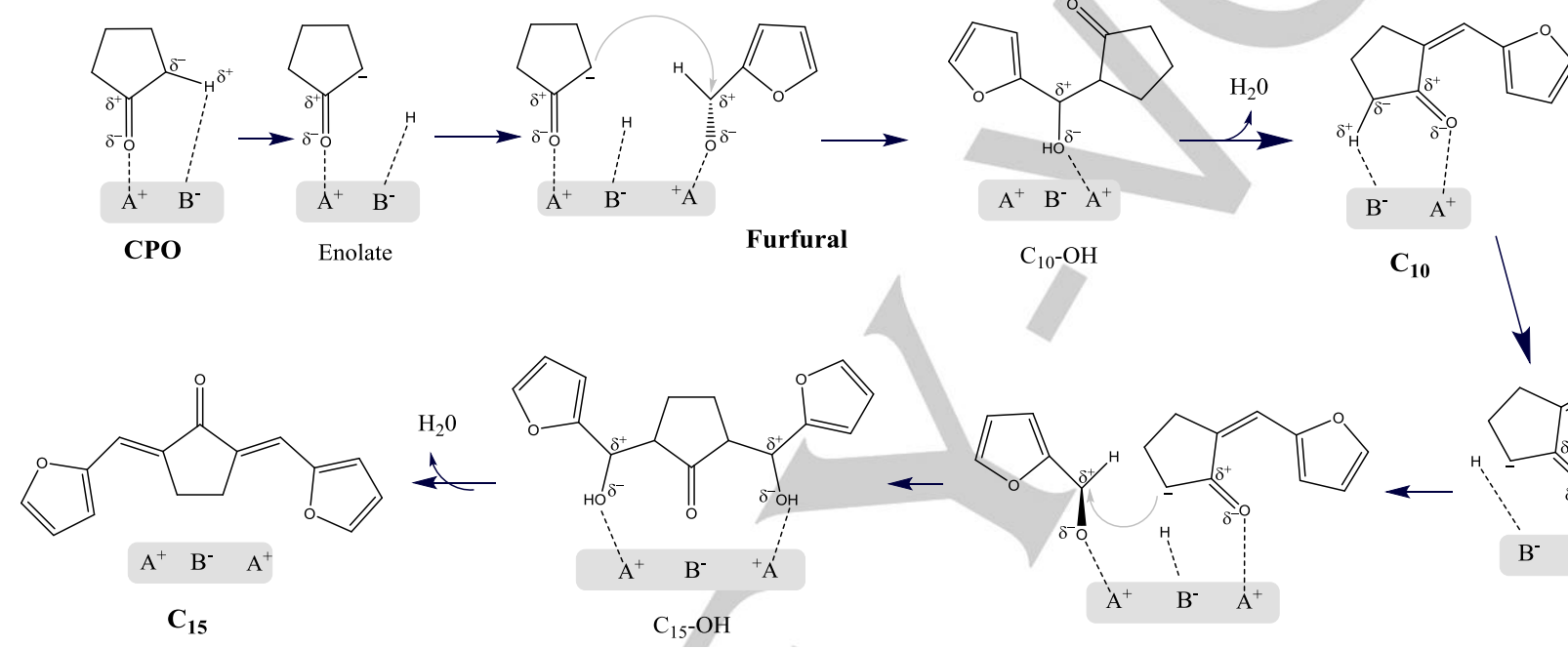

Scheme 1. Main products obtained in the furfural - cyclopentanone condensation catalyzed by $\mathrm{Mg}-\mathrm{Zr}$

TG analysis of the spent catalyst after the extraction with ethyl acetate is also plotted in Fig. 2 with the aim to analyze the effectivity of the extraction with the organic solvent. Signals related to the $\mathrm{C} 10$ and $\mathrm{C} 15$ condensation adducts are still detected, with very low intensity, which can justify the carbon balance values lower than $100 \%$. The most highlighting point in this analysis is, however, the almost complete disappearance of the peak at $713 \mathrm{~K}$. The high content in polar groups of this intermediate justifies its higher solubility in ethyl acetate, obtaining a much more effective extraction. On the other hand, and being congruent with the GC-FID analyses, no evidences of any peak related to the CPO self-condensations were observed, without any peak around 620 or $562 \mathrm{~K}$ (theoretical temperatures of the corresponding dimer and trimer CPO self-condensation adducts).

Analyzing all the results, many different reasons justify the choice of $303 \mathrm{~K}$ as the optimum temperature: at these conditions, the global yield obtained is the maximum as well as the $\mathrm{C} 15 / \mathrm{C} 10$ ratio. Besides, best carbon balance corresponds to these conditions, with a good equilibrium between too strong adsorption of $\mathrm{C} 15$ intermediates and not too much deposition of solid compounds. The initial conversion rate on a mass basis, according to the equation 1 , was calculated in order to compare cyclopentanone and acetone, respectively).

Considering that previous results suggest that the reaction can be limited by the complete conversion of furfural, the influence of different initial reactant ratios was also studied in the reaction at $303 \mathrm{~K}$. Main results after 4 hours are summarized in Table 2 , whereas the temporal profiles are attached as supplementary information (Figure S3). Results obtained allow discarding the existence of equilibrium limitations, since complete conversion of cyclopentanone was observed when working in furfural excess. This results contrast with the clear equilibrium observed for the aldol condensation of other biomass-derived compounds. ${ }^{[16]}$ However, it is congruent with the higher stability of the condensation adducts produced by the higher stability of the CPO derived enolate intermediate and the higher molecular mass of the condensation adducts, hindering the presence retroaldolization reactions. This fact justifies the higher reaction rate of this reaction, in comparison with the initial rate obtained with the acetone. Carbon balances higher than $84 \%$ were obtained in all the cases and this parameter increases at increasing furfural concentration, suggesting that no side reactions are detected and discarding also the reaction between two molecules of furfural. These side-reactions, known as 
Cannizzaro $^{[23]}$ reactions have been previously suggested (but working at different temperatures) and they would produce 2-furanic acid and furfuryl alcohol. As it could be expected, the increase in the furfural-cyclopentanone ratio implies a decrease in the relative selectivity to the dimer, in such a way that at 10:1 less than $1 \%$ of the $\mathrm{C} 10$ was observed. However, these conditions strongly limit the reaction, being the $\mathrm{C} 15$ yield twice lower than the value obtained working with a moderate excess of furfural (3:1 and 5:1 conditions).

According to the experimental results, the reaction mechanism suggested is summarized in Scheme 1, being similar to the most accepted network of other condensation between biomass derived compounds, such as furfural and acetone. According to this, reaction begins with abstraction of the $\alpha$-proton of cyclopentanone, when this molecule is adsorbed on a medium-strength basic site of $\mathrm{Mg}-\mathrm{Zr}$, and the subsequent interaction with one molecule of furfural previously adsorbed on the catalytic surface, obtaining the precursor or the first intermediate. The hydroxyl group of this molecule suffers dehydration because of the presence of acid sites, resulting in the first condensation adduct (C10) that is clearly detected by GC-FID in its cis and trans stereoisomers. However, by contrast with what happened in the condensation of furfural and acetone, this dehydration is not so fast and the intermediate is also detected also with its two isomers. In the experiments, these compounds have a typical profile of a first product in a serial reaction, reaching a maximum after which its yield fast decreases because of the formation of the $\mathrm{C} 15$ condensation adducts. Considering that there are two $\alpha$-protons, a second condensation is also possible, obtaining the $\mathrm{C} 15$ alcohol and the subsequent $\mathrm{C} 15$ condensation adduct after the dehydration (with its three combinations: cis-cis, cis-trans, and trans-trans). In this case, the dehydration is faster and the adsorption more relevant, in such a way that only small signals of hydroxyl compounds are detected (always lower than $5 \%$ of the total amount of C15's).

Despite the fact that the insolubility of both products prevents a complete kinetic study, profiles of both reactants suggest a first order reaction respect to the cyclopentanone, in good agreement with previous studies and congruent with the proposed mechanism. The absence of CPO self-condensation products, to the contrary of what is reported in reaction homogeneously catalyzed, ${ }^{[14 a]}$ indicates the high affinity between $\mathrm{FFL}$ and the catalytic surface, as it was previously demonstrated for other reactions catalyzed by $\mathrm{Mg}-\mathrm{Zr} .{ }^{[16]}$ This hypothesis is congruent with a zero order dependence on the furfural, as it

Table 2. Results after 4 hours of condensation between furfural and cyclopentanone at $303 \mathrm{~K}$ catalyzed by $0.1 \mathrm{~g}$ of $\mathrm{Mg}-\mathrm{Zr}$ as function of the reactant ratio ${ }^{[a]}$

\begin{tabular}{cccccc}
\hline FFL:CPO & $\begin{array}{c}\text { Conv. FFL } \\
(\%)\end{array}$ & $\begin{array}{c}\text { Conv. CPO } \\
(\%)\end{array}$ & $\begin{array}{c}\Psi_{\mathrm{C} 10} \\
(\%)\end{array}$ & $\begin{array}{c}\Psi_{\mathrm{C} 15} \\
(\%)\end{array}$ & $\begin{array}{c}\text { C.B. } \\
(\%)\end{array}$ \\
\hline $1: 1$ & 98.3 & 66.4 & 31.7 & 34.4 & 83.8 \\
$3: 1$ & 67.3 & 97.9 & 13.7 & 49.6 & 88.4 \\
$5: 1$ & 51.1 & 99.2 & 2.37 & 53.7 & 97.0 \\
$10: 1$ & 26.2 & 98.9 & 0.67 & 29.1 & 97.1 \\
\hline
\end{tabular}

[a] The mathematical expressions of atomic yields and carbon balance are detailed in the experimental section. happened with the acetone. ${ }^{[16]}$

In conclusion, promising results for the furfural and cyclopentanone aldol condensation are obtained working at mild conditions and using $\mathrm{MgZr}$ as heterogeneous catalyst. Almost complete conversion of furfural in 4 hours was obtained is spite of the reaction conditions tested, obtaining two main results: Maximum conversion was obtained working at $303 \mathrm{~K}$ with an excess of furfural (3:1 initial molar rate), with $67 \%$ of FFL conversion and almost $100 \%$ of CPO. At these conditions, more than $60 \%$ of final yields are obtained, with almost $50 \%$ of the main interesting adduct (C15). However, if the objective is to maximize the $\mathrm{C} 15$ yield, optimum results were obtained with more furfural excess (5:1). Both adducts (C10 and C15) are obtained selectively (no side reactions, neither oligomerizations nor CPO self condensation) and perfectly separated from the reagents as a solid phase. These results indicate a higher activity than using acetone as linking molecule. The evolution of reactants profiles is congruent with a first order reaction mechanism of two serial steps, in good agreement with the mechanism of other similar condensations. The highest stability of both adducts in the solid phase justifies the absence of retro aldolization.

\section{Experimental Section}

$\mathrm{Mg}$-Zr mixed oxide was synthesized according to the sol-gel procedure previously optimized and reported in our previous work ${ }^{[16]}$. The gel is obtained from the solution of the corresponding nitrate salts when $\mathrm{pH}$ is increased to 10 . Gel is aged for 24 hours, filtered, washed and dried before the heat treatment in $\mathrm{He}$ flow, using a $5 \mathrm{~K} / \mathrm{min}$ rate to $873 \mathrm{~K}$, holding these conditions for 3 hours. Reactions are carried out in a $0.5 \mathrm{~L}$ autoclave reactor (Autoclave Engineers EZE Seal) equipped with a PID controller. $0.25 \mathrm{~L}$ of an aqueous solution of both reagents was used (percentage of organic compounds and FFL/CPO ratio specified in each experiment). $100 \mathrm{mg}$ of $\mathrm{Mg}-\mathrm{Zr}$ is added as catalyst (particle diameter of 50-80 $\mu \mathrm{m}$ ). Air is purged with $\mathrm{N}_{2}$ for three times and reaction is carried out at 10 bar of $\mathrm{N}_{2}$ under $1000 \mathrm{rpm}$ of stirring. The reaction evolution is studied by analysing different aqueous samples collected from the sampling port. These samples were filtered to prevent any solid particle and extracted in ethyl acetate (volume ratio of 1:1) before being analysed by gas chromatography with a FID detector (Shimadzu GC-2010, using a $30 \mathrm{~m}$ long CP-Sil 8CB capillary column). Two different techniques were used for the compounds identification: gas chromatography with a mass spectroscopic detector, GC-MS (QP2010 Plus Instrument), using a $30 \mathrm{~m}$ long TRB-5MS column; and the nuclear magnetic resonance (Brukner AV600) programmed with frequencies of 600.15 and $150.91 \mathrm{~Hz}$ for $\mathrm{H}$ and $\mathrm{C}$, respectively, using deuterated chloroform. The obtained spectra were compared with the theoretical values of chemical shift (it allows the identification of the product) and J-coupling (determines the optical isomers). The calibrations of furfural, cyclopentanone and the first adduct (C10) are carried out using commercial samples (Sigma Aldrich, >99\%) whereas the C15's calibration is done using the relative carbon concept proposed by Scanlon. ${ }^{[24]}$ Results are shown in the manuscript using the conversion, atomic yields and carbon balance concepts defined as follows (Eq. 2-4):

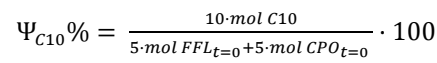

$$
\begin{aligned}
& \Psi_{C 15} \%=\frac{15 \cdot \mathrm{mol} \mathrm{C} 15}{5 \cdot \mathrm{mol} \mathrm{FFL}_{t=0}+5 \cdot \mathrm{mol} \mathrm{CPO}_{t=0}} \cdot 100 \\
& \text { C.B. }(\%)=\frac{5 \cdot \mathrm{mol} \mathrm{FFL}+5 \cdot \mathrm{mol} \mathrm{CPO}+10 \cdot \mathrm{mol} \mathrm{C} 10_{\mathrm{liq}+\mathrm{sol}}+15 \cdot \mathrm{mol} \mathrm{C5} 5_{\mathrm{liq}+\mathrm{sol}}}{5 \cdot \mathrm{mol} \mathrm{FFL} t=0+5 \cdot \mathrm{mol} \mathrm{CPO}_{t=0}} \cdot 100
\end{aligned}
$$


Once the reaction is completed, the obtained solid yellow-colored product was filtered, dried, weight and analyzed by GC-FID after complete solution in ethyl acetate.

\section{Acknowledgements}

This work was supported by the Spanish Ministry for Economy and and Competitiveness (MINECO) (contract CTQ2014-52956C3-1-R).

Keywords: aldol reaction $\bullet$ biofuels $\cdot \mathrm{C}-\mathrm{C}$ coupling $\cdot$ mixed oxides $\cdot \mathrm{MgZr}$

[1] a) D.M. Alonso, J.Q. Bond, J.A. Dumesic, Green Chem. 2010, 9, 1493 1513; b) K. Wu, Y. Wu, Y. Chen, H. Chen, J. Wang, M. Yang, ChemSusChem 2016, 9, 1355-1385; c) L. Faba, E. Díaz and S. Ordóñez, Renew. Sust. Energ. Rev. 2015, 51, 273-287.

[2] G. W. Huber, J. N. Chheda, C. J. Barrett, J. A. Dumesic, Science 2005 308, 1446-1450

[3] H. Olcay, A.V. Subrahmanyam, R. Xing, J. Lajoie, J.A. Dumesic, G.W. Huber, Energ. Environ. Sci. 2013, 6, 205-216.

[4] a) L. Faba, E. Díaz, S. Ordóñez, Appl. Catal. B 2012, 113, 201-211; b) L. Faba, E. Díaz, S. Ordóñez, ChemSusChem 2013, 6, 463-473.

[5] R. Selvin, G. R. Rajarajeswari, L. Selva Roselin, V. Sadasivam, B. Sivasankar, K. Rangaraj, Appl. Catal. A 2001, 219, 125-129.

[6] C. S. Bîldea, I. Patraşcu, J. G. Segovia Hernández, A. A. Kiss, Comput. Aided Chem. Eng. 2016, 38, 979-984.

[7] W. Shen, G. A. Tompsett, K. D. Hammond, R. Xing, F. Dogan, C. P. Grey, W. C. Conner Jr, S. M. Auerbach, G. W. Huber, Appl. Catal. A 2011, 392, 57-68.

[8] B. Danon, W. Hongsiri, L. van der Aa, W. de Jong, Biomass Bioenerg. 2014, 66, 364-370

[9] a) J. Guo, G. Xu, Z. Han, Y. Zhang, Y. Fu, Q. Guo, ACS Sustain. Chem Eng. 2014, 2, 2259-2266; b) X. L. Li, J. Deng, J. Shi, T. Pan, C. G. Yu, H. J. Xu, Y. Fu, Green Chem. 2015, 17, 1038-1046.

[10] M. Hronec, K. Fulajtarova, I. Vavra, T. Sotak, E. Dobrocka, M. Micusik, Appl. Catal. B 2016, 181, 210-219.

[11] a) J. Xu, J. Cao, Q. Ma, X. Peng, Asian J. Chem. 2013, 25, 3847-3849; b) D. Liang, G. Li, Y. Liu, J. Wu, X. Zhang, Catal. Commun. 2016, 81, 33-36.

[12] B. Rajesh Kumar and S. Saravanan, Renew. Sust. Energ. Rev. 2016 , 60, 84-115.

[13] M. Mascal, S. Dutta, I. Gandarias, Angew. Chem. 2014, 126, 18851888.

[14] a) M. Hronec, K. Fulajtarova, T. Liptaj, M. Stolcova, N. Pronayova, T. Sotak, Biomass Bioenerg. 2014, 63, 291-299; b) Q. Deng, J. Xu, P. Han, L. Pan, L. Wang, X. Zhang, J. Zou, Fuel Process. Technol. 2016 148, 361-366

[15] L. Hora, O. Kikhtyanin, L. Čapek, O. Bortnovskiy, D. Kubička, Catal. Today 2015, 241, 221-230

[16] L. Faba, E. Díaz, S. Ordóñez, Appl. Catal. B 2012, 113, 201-211.

[17] D. Liang, G. Li, Y. Liu, J. Wu, X. Zhang, Catal. Commun. 2016, 81, 3336.

[18] a) L. Faba, E. Díaz, S. Ordóñez, Appl. Catal. B 2012, 113, 201-211; b) V. K. Díez, C. R. Apesteguía, J. I. Di Cosimo, J. Catal. 2006, 240, 235 244. c) S. Ordóñez, E. Díaz, M: León, L. Faba, Catal. Today 2011, 167 $71-76$

[19] a) R.R. O'Neill, L. Vauoye, C.D. Bellefou, F.Ayouache, Appl. Catal. B 2014, 144, 46-56; b) L. Faba, E. Díaz, S. Ordóñez, Biomass Bioenerg. 2013, 56, 592-599.
[20] a) L. Faba, E. Díaz, S. Ordóñez, Appl. Catal. B 2012, 113, 201-211; b) L. Hora, V. Kelbichová, O. Kikhtyanin, O. Bortnovskiy, D. Kubicka, Catal. Today 2014, 223, 138-147.

[21] S.E. Stein, R.L. Brown, J. Chem. Inf. Model. 1994, 34, 581-587.

[22] J. Cueto, L. Faba, E. Díaz, S. Ordóñez, Appl. Catal. B 2017, 201, 221 231.

[23] B. Arora, P.S. Pandey, M.N. Gupta, Tetrahedron Lett. 2014, 55, 39203922

[24] J.T. Scanlon, D.E. Willis, J. Chromatogr. Sci. 1985, 23, 333-340. 


\section{COMMUNICATION}

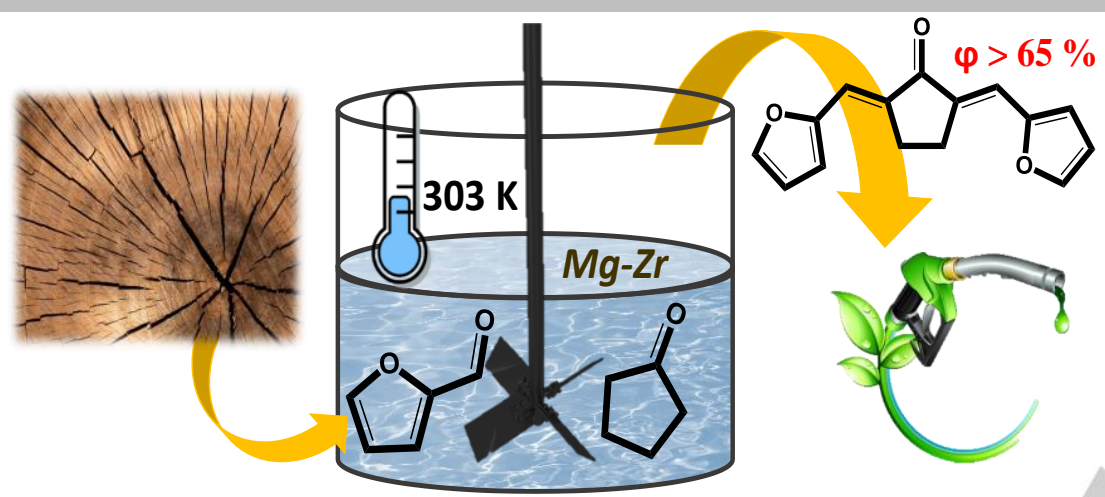

J. Cueto, L. Faba, E. Díaz, S. Ordóñez*

Page No. - Page No.

Cyclopentanone as alternative linking reactant for heterogeneously catalysed furfural aldol condensation

Insert the text of table of contents 